\title{
Dispositivos de Administración Electrónica de Nicotina y sus Efectos en los Tejidos Periodontales y la Región Orofacial: Revisión de la Literatura
}

\author{
Electronic Nicotine Delivery Systems and their Effect in \\ Periodontal Tissue and the Orofacial Region. A Review
}

María José Zilleruelo Pozo'; Paula Riera Sanz² \& Gianitsa Georgudis Pinto²

ZILLERUELO, P. M. J.; RIERA, S. P. \& GEORGUDIS, P. G. Dispositivos de administración electrónica de nicotina y sus efectos en los tejidos periodontales y la región orofacial: Revisión de la Literatura. Int. J. Odontostomat., 12(3):287-295, 2018.

RESUMEN: Los dispositivos de administración electrónica de nicotina aparecieron con el objetivo de reemplazar el uso del tabaco y contribuir al reciente crecimiento de las políticas antitabaco. Actualmente, los efectos que producen los químicos que contiene son desconocidos. El objetivo de este trabajo consistió en describir la información encontrada en la literatura sobre los posibles daños generados por los dispositivos de administración electrónica de nicotina, en la cavidad bucal y los tejidos periodontales. Se realizó una búsqueda electrónica y manual, sin límite de idioma ni año. Se excluyeron, opiniones de expertos y artículos que estudiaran otros dispositivos de administración de nicotina. Se seleccionaron 18 artículos, 7 estudios in-vitro, 2 estudios longitudinales, 2 ensayos clínicos y 7 reportes de caso. Se encontró que existen niveles de citotoxicidad elevados, observando cambios a nivel de morfología y metabolismo celular. Sin embargo, si lo comparamos con el tabaco, se observa que los niveles de toxicidad son menores en los dispositivos de administración electrónica de nicotina. En los estudios longitudinales y ensayos clínicos se observó un aumento del sangramiento al sondaje y de la circulación sanguínea, medida con láser Doppler, al cambiar de cigarro convencional a cigarro electrónico. Los reportes de caso informan de importantes traumatismos en el territorio maxilofacial, causados por la explosión de estos dispositivos, durante su uso. Los dispositivos de administración electrónica de nicotina son tóxicos a nivel de las células periodontales, generando necrosis y daños al ADN celular. Presentan riesgos de uso, reportándose traumatismos graves a nivel oral y maxilofacial, por sobrecalentamiento de las baterías. Los ensayos clínicos y estudios longitudinales no fueron concluyentes, por lo que se debe seguir investigando en la materia.

PALABRAS CLAVE: Sistemas electrónicos de liberación de nicotina, enfermedades periodontales, cavidad oral.

\section{INTRODUCCIÓN}

Los dispositivos de administración electrónica de nicotina se definen como aquellos equipos operados mediante baterías de litio que al calentar una solución producen un aerosol, el cual es liberado e inhalado por el usuario (Born et al., 2015). Este proceso se activa mediante la aspiración realizada por el usuario, lo cual produce un cambio de presión que estimula un sensor, el cual le indica al atomizador que empiece a aumentar la temperatura, sin producir combustión, llegando a alcanzar temperaturas entre 100$250{ }^{\circ} \mathrm{C}$ (Kanodia, 2016).
Estos dispositivos agrupan tanto al cigarro electrónico como a los vaporizadores. Se introdujeron al mercado el año 2007 en Estados Unidos (Born et al.) y según la OMS su consumo se ha duplicado en los últimos cinco años, existiendo hoy más de 400 marcas disponibles (Chen, 2013). En el año 2010 solo un $0,6 \%$ de los adultos a nivel mundial lo había probado al menos 1 vez; en 2011 un 6,2 \% y para el año 2013 un 8,1 \% de la población mundial (Born et al.).

\footnotetext{
${ }^{1}$ Cirujano Dentista, Universidad de los Andes, Santiago, Chile.

${ }^{2}$ Cirujano Dentista, Especialista en Periodoncia, Universidad de los Andes, Santiago, Chile.
} 
Se ha descrito que la solución contenida en los dispositivos presenta múltiples químicos posiblemente dañinos para la salud como propilenglicol, glicerol y otros agentes, además de nicotina en el $99 \%$ de los casos (Marynak et al., 2017). Esta última se ha asociado a estrés oxidativo y disminución de la respuesta inmune, llevando finalmente a apoptosis celular y afectando la reparación de los tejidos (Sancilio et al., 2017). A su vez se han encontrado agentes carcinógenos, que también están presentes en el tabaco, como nitrosaminas tabaco-específicas (TSNA) (Kim \& Shin, 2013), aldehídos, compuestos carbonilos, metales, componentes volátiles orgánicos, compuestos fenólicos, hidrocarbonos aromáticos poli-acrílicos, alcaloides del tabaco, solventes, donde algunas de estas sustancias están aprobadas por la Food \& Drug Administration (FDA), pero no para ser inhaladas (Ebbert et al., 2015).

Además, se han reportado eventos adversos, principalmente complicaciones pulmonares, como neumonía, quemaduras y explosiones de las baterías de litio causando graves daños en los usuarios. Esto se da por un proceso denominado «thermal runaway", que se ha descrito en la literatura como una retroalimentación positiva descontrolada del aumento de la temperatura, que puede terminar en combustión (Wang et al., 2012).

En Chile estos dispositivos están regulados desde el 2010, con la modificación de la Ley de Tabaco (Ley 20.660, 2013). Esto tiene como propósito una mayor fiscalización de la venta y distribución de estos productos. Además, se clasificó como un producto farmacéutico, al contener nicotina la cual es una droga tóxica para los seres humanos (Ley 20.660).

Nos referiremos a los dispositivos de administración electrónica de nicotina también como cigarro electrónico, agrupando en este término a los vaporizadores. No se hará diferencia entre ambos debido a que su funcionamiento y composición interna es prácticamente la misma.

El objetivo primario de esta revisión es describir los efectos de los dispositivos de administración electrónica de nicotina sobre los tejidos periodontales. Como objetivo secundario se busca describir la comparación de los efectos a nivel periodontal entre los dispositivos electrónicos y el cigarro convencional, además de reportar los casos de trauma dento-facial asociados a su uso.

\section{MATERIAL Y MÉTODO}

Se realizó la siguiente estrategia de búsqueda utilizando palabras clave, combinadas con los términos booleanos AND y OR. La búsqueda bibliográfica se realizó en cinco bases de datos: EBSCO, Pubmed, Trip Database, Epistemonikos y The Cochrane Library:

(Periodontal OR "periodontal disease" OR mouth OR oral OR "Periodontal Diseases"[Mesh]) AND ("Electronic Nicotine Delivery Systems"[Mesh] OR "electronic cigarettes" OR "e-cigarette" OR "e-smoking" OR vaping).

Los criterios de inclusión fueron sin límite de idioma y año, estudios in vitro e in-vivo, ensayos clínicos, estudios observacionales, revisiones sistemáticas y reportes de caso. Se excluyeron opiniones de expertos, estudios sobre fumadores pasivos, reportes sobre ingestión del líquido de los dispositivos electrónicos de administración de nicotina, estudios sobre otros dispositivos de administración de nicotina (no electrónicos), estudios sobre efectividad para dejar el hábito tabáquico y percepción de los usuarios sobre su uso.

Se evalúo la calidad de reporte utilizando las pautas STROBE, para estudios observacionales; CONSORT para ensayos clínicos, CRIS para estudios in vitro y CARE para reportes de caso. El nivel de evidencia científica se evaluó según las pautas del CEBM de la Oxford University.

El riesgo de sesgo se evaluó para los estudios observacionales y ensayos clínicos, utilizando el método publicado por The Cochrane Collaboration. Según esto, se consideró a aquellos trabajos con una puntuación 6 , como bajo riesgo de sesgo, moderado con puntuación 4 ó 5 y alto de 3 a 0 puntos.

\section{RESULTADOS}

Los estudios fueron descartados siguiendo los criterios de inclusión y exclusión previamente mencionados.

En la búsqueda manual retrógrada se encontraron 11 artículos nuevos, de los cuales solo dos quedaron seleccionados luego del descarte. Así quedaron seleccionados 18 artículos en total, los cuales constituyen dos ensayos clínicos, dos estudios 
Tabla I. Estudios seleccionados para la revisión.

\begin{tabular}{|c|c|c|c|}
\hline Título & Autores & Año & Tipo Artículo \\
\hline $\begin{array}{l}\text { Monitoring nicotine intake from e-cigarettes: } \\
\text { measurement of parent drug and metabolites in oral } \\
\text { fluids. }\end{array}$ & Papaseit et al. & 2016 & $\begin{array}{l}\text { Ensayo clínico controlado } \\
\text { aleatorizado, tipo cross-over }\end{array}$ \\
\hline $\begin{array}{l}\text { A Pilot Study of the gingival response when smokers } \\
\text { switch from smoking to vaping. }\end{array}$ & Wadia et al. & 2016 & Estudio Longitudinal (Piloto) \\
\hline $\begin{array}{l}\text { Modifications in Human Oral Fibroblast Ultrastructure, } \\
\text { Collagen Production and Lysosomal Compartment in } \\
\text { response to E-Cigarette fluids. }\end{array}$ & Sancilio et al. & 2017 & Estudio in vitro \\
\hline $\begin{array}{l}\text { Influence of E-smoking liquids on Human Periodontal } \\
\text { Ligament Fribroblasts. }\end{array}$ & Willershausen et al. & 2014 & Estudio in vitro \\
\hline $\begin{array}{l}\text { E-Cigarette vapor induces an apoptotic responde in } \\
\text { human gingival epithelial cells through the caspase- } 3 \\
\text { pathway. }\end{array}$ & Rouabhia et al. & 2017 & Estudio in vitro \\
\hline $\begin{array}{l}\text { Electronic cigarettes induce DNA stand breaks and cell } \\
\text { death independently of nicotine in cell lines. }\end{array}$ & Yu et al. & 2015 & Estudio in vitro \\
\hline $\begin{array}{l}\text { Characterization of Electronic Cigarette Aerosol and Its } \\
\text { Induction of Oxidative Stress Response in Oral } \\
\text { Keratinocytes. }\end{array}$ & Ji et al. & 2016 & Estudio in vitro \\
\hline $\begin{array}{l}\text { Citotoxicity and apoptosis induction by e-cigarette fluids } \\
\text { in human gingival fibroblasts. }\end{array}$ & Sancilio et al. & 2016 & Estudio in vitro \\
\hline $\begin{array}{l}\text { Crosstalk between oral and general health status in e- } \\
\text { smokers. }\end{array}$ & Tatullo et al. & 2016 & Estudio Longitudinal (Piloto) \\
\hline $\begin{array}{l}\text { Do you mind if I vape? Immediate effects of electronic } \\
\text { cigarettes on perfusion in buccal mucosal tissue - a pilot } \\
\text { study. }\end{array}$ & Reuther et al. & 2016 & $\begin{array}{l}\text { Ensayo clínico no controlado } \\
\text { estudio piloto }\end{array}$ \\
\hline $\begin{array}{l}\text { E-cigarettes and flavourings induce inflammatory and } \\
\text { prosenescence responses in oral epithelial cells and } \\
\text { periodontal fibroblasts. }\end{array}$ & Sundar et al. & 2016 & Estudio in vitro \\
\hline $\begin{array}{l}\text { Electronic cigarette explosion: case report of an } \\
\text { emerging cause of orofacial trauma. }\end{array}$ & Chu et al. & 2016 & Reporte de caso \\
\hline $\begin{array}{l}\text { Oral Trauma and tooth avulsion following explosion of e- } \\
\text { cigarette. }\end{array}$ & Roger et al. & 2016 & Reporte de caso \\
\hline Electronic cigarette explosion injuries: A case series. & Kumetz et al. & 2016 & Reporte de caso \\
\hline $\begin{array}{l}\text { Electronic cigarette explosion associated with extensive } \\
\text { intraoral injuries. }\end{array}$ & Brooks et al. & 2016 & Reporte de caso \\
\hline Electronic cigarette explosions involving the oral cavity. & Harrison \& Hicklin & 2016 & Reporte de caso \\
\hline $\begin{array}{l}\text { E-cigarette Blast Injury: Complex Facial Fractures and } \\
\text { Pneumocephalus. }\end{array}$ & Arrchambeau et al. & 2016 & Reporte de caso \\
\hline $\begin{array}{l}\text { Electronic cigarette explosion resulting in a } \mathrm{C} 1 \text { and } \mathrm{C} 2 \\
\text { fracture: A Case Report. }\end{array}$ & Norii \& Plate & 2016 & Reporte de caso \\
\hline
\end{tabular}

longitudinales, siete estudios in vitro y siete reportes de caso (Tabla I). Solo en The Cochrane Library no se encontraron resultados respecto al tema.

El riesgo de sesgo de los estudios longitudinales y ensayos clínicos se específica en la Tabla II.
Cabe mencionar que la mayoría de los estudios considerados representan bajo nivel de evidencia, sin embargo, fueron considerados debido a que constituían la mejor evidencia disponible con respecto al tema.

Tabla II. Evaluación riesgo de sesgo según The Cochrane Collaboration

\begin{tabular}{|c|c|c|c|c|c|}
\hline Autor & $\begin{array}{c}\text { Tamaño de la } \\
\text { muestra }\end{array}$ & Aleatorización & $\begin{array}{c}\text { Ocultamiento de la } \\
\text { a signación }\end{array}$ & Pérdidas & Riesgo de Sesgo \\
\hline Wadia et al., (2016) & 1 & 1 & 0 & 1 & Alto \\
\hline Tatullo et al., (2016) & 1 & 1 & 1 & 1 & Moderado \\
\hline Papaseit et al., (2016) & 1 & 0 & 0 & 1 & Alto \\
\hline Reuther et al., (2016) & 1 & 0 & 0 & 1 & Alto \\
\hline
\end{tabular}




\section{DISCUSIÓN}

Los siete estudios in vitro que fueron incluidos analizan la citotoxicidad, ya sea del líquido o del aerosol producido por los dispositivos de administración electrónica de nicotina (Tabla III). Todos los trabajos utilizaron algún saborizante con nicotina adicionada, en distintas concentraciones. Sancilio et al. (2016) menciona que $1 \mathrm{mg} / \mathrm{ml}$ es la concentración que se encuentra en la saliva de la mayoría de los fumadores de cigarro convencional y Ji et al. (2016) determinó que la toxicidad o el daño producido por estos dispositivos era dosis-dependiente, lo cual está directamente relacionado con la intensidad y la duración de la aspiración del cigarro electrónico. Esto es de gran importancia, ya que los efectos y la variabilidad producida entre los distintos usuarios es bastante amplia, dependiendo del modo de uso (Ramôa et al., 2017).

En cuanto al formato empleado, Sancilio et al. (2016, 2017) y Willershausen et al. (2014) utilizaron el compuesto líquido y embebieron las células en la solución. Mientras que Rouabhia et al. (2017), Yu et al. (2016), Ji et al. y Sundar et al. (2016), mediante una cámara de humo, lograron producir un aerosol el cual impregnaba a las células. En los dos trabajos realizados por Sancilio et al. (2016, 2017), el líquido se calentó previamente y se observó una mayor toxicidad. Se cree que al aumentar la temperatura se altera la composición del líquido, generándose nuevas partículas desde el atomizador. Se puede deducir que aquellos estudios que utilizaron aerosol tienen mayor predictibilidad en cuanto al efecto que producirán en los tejidos orales, debido a que las partículas no son iguales en estado líquido.

En cuanto a la morfología celular, Rouabhia et al. observaron en el microscopio invertido que las células expuestas al aerosol se encontraban de mayor tamaño y elongadas, reduciéndose a la vez la densidad celular en el campo. Sancilio et al. (2016, 2017) observaron mayor número de vacuolas vacías en citoplasma, retículo endoplasmático rugoso alargado, desorganizado y en la periferia, además de una mitocondria difícilmente distinguible. Todos estos son signos de citotoxicidad a nivel estructural, sin embargo, en estos últimos estudios se determinó que esto solo ocurría en el grupo con nicotina, no así en el que se exponía solo al saborizante.

Rouabhia et al. señalaron que el aerosol del cigarro electrónico determina una menor viabilidad celular de forma estadísticamente significativa, observándose un aumento de células tanto necróticas como apoptóticas, debido a que ocurre fragmentación del ADN celular y aumento de enzimas proapoptóticas, como la caspasa 3. De la misma forma, Yu et al. establecieronque el aerosol del cigarro electrónico produce un aumento en la rotura de doble hebra del ADN, también de forma estadísticamente significativa para las células expuestas a aerosol, con y sin nicotina. Además, señala un aumento de apoptosis y necrosis celular, ya que se produce detención del ciclo celular.

Sundar et al. evaluaron solo la presencia de marcadores de daño de ADN y comprobó que estaban aumentados de forma estadísticamente significativa en los grupos expuestos al aerosol. Sancilio et al. (2016) también concluyeron que hay una disminución en la viabilidad celular y mayor expresión de proteínas pro-apoptóticas, específicamente la proteína BAX, siendo también independiente de si contenían nicotina o no.

Sundar et al. afirmaron que se producen citoquinas pro-inflamatorias y aumentan los niveles de ciclooxigenasa 2, prostaglandina E2 y especies reactivas de oxígeno, llevando a estrés oxidativo, el que se ha relacionado directamente con mayor respuesta inflamatoria específicamente a nivel gingival (Ito et al., 2012). En cambio, los resultados del estudio de Ji et al. no fueron concluyentes.

Willershausen et al. evaluaron los niveles de ATP y propone que existe disminución en la viabilidad celular al observarse una disminución estadísticamente significativa de la cantidad de ATP celular, para el grupo con y sin nicotina.

Podríamos atribuir la toxicidad de los cigarros electrónicos a la nicotina, sin embargo, algunos de los estudios in vitro incluidos en esta revisión señalan que esta toxicidad no recae solamente en ésta. Willerhausen et al. y Yu et al., establecen que la citotoxicidad es independiente de si tienen o no nicotina, ya que ambos grupos presentaban toxicidad elevada de forma estadísticamente significativa, comparada con el grupo control. Esto se puede atribuir a los saborizantes (Willershausen 


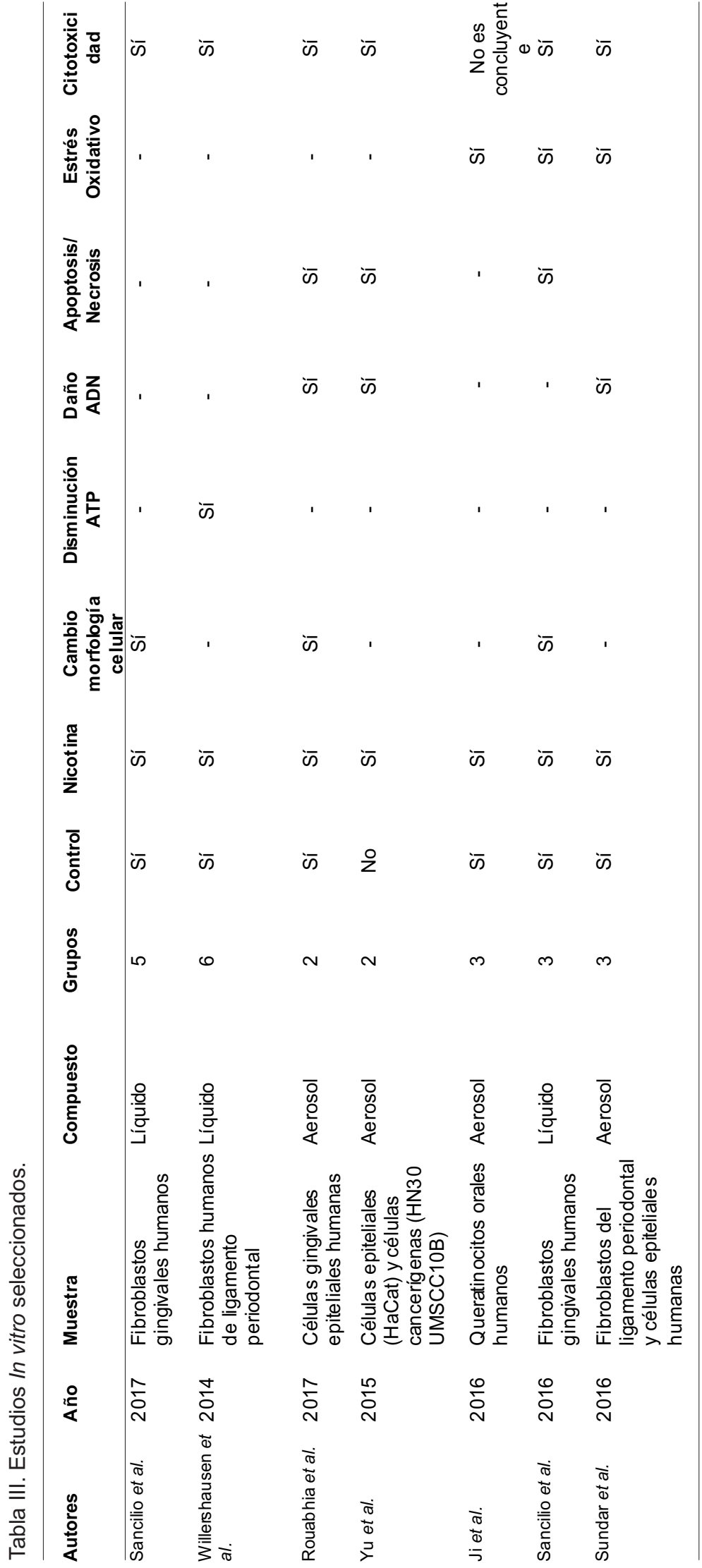

et al., 2014; Sundar et al.) especialmente, el sabor mentol que genera una respuesta inflamatoria más marcada en los tejidos, al presentar una mayor cantidad de partículas en el aerosol (Ji et al.). Entonces podemos decir que la toxicidad y el daño inducido por el cigarro electrónico no es solamente atribuible a la presencia de nicotina, sin perjuicio de que en aquellos grupos con nicotina se observa un efecto aún más marcado en los tejidos.

En comparación con el cigarro convencional los dispositivos de administración electrónica de nicotina presentan menor citotoxicidad a nivel de los tejidos en general, pero igual generan daño, solo que en menor medida. Yu et al. compararon los efectos del aerosol del cigarro electrónico con el convencional, observando que donde ocurría la mayor cantidad de daño al ADN celular era en el grupo expuesto a tabaco $(0,5 \mathrm{mg}$ de nicotina). El estudio de Ji et al. observaron que el sabor mentol generaba efectos similares al grupo expuesto a tabaco. Entonces si bien los efectos producidos por el cigarro convencional son más marcados, el aerosol del cigarro electrónico es citotóxico para las células epiteliales humanas, especialmente con algunos saborizantes como el mentol.

Wadia et al. (2016) y Tatullo et al. (2016) evaluaron los parámetros clínicos periodontales luego de cambiar de cigarro convencional a electrónico, siendo ambos estudios longitudinales pequeños con poco seguimiento. Plantean que existe un aumento estadísticamente significativo en el sangramiento de los pacientes al utilizar cigarro electrónico, observando mejorías en el índice de placa y un cese en el enmascaramiento de la inflamación (Tatullo et al.). Lo anterior muestra que tanto dejar el cigarro convencional como utilizar los dispositivos electrónicos, tendría efectos similares en los tejidos orales.

Reuther et al. (2016) realizaron un ensayo clínico donde se midió el flujo san- 


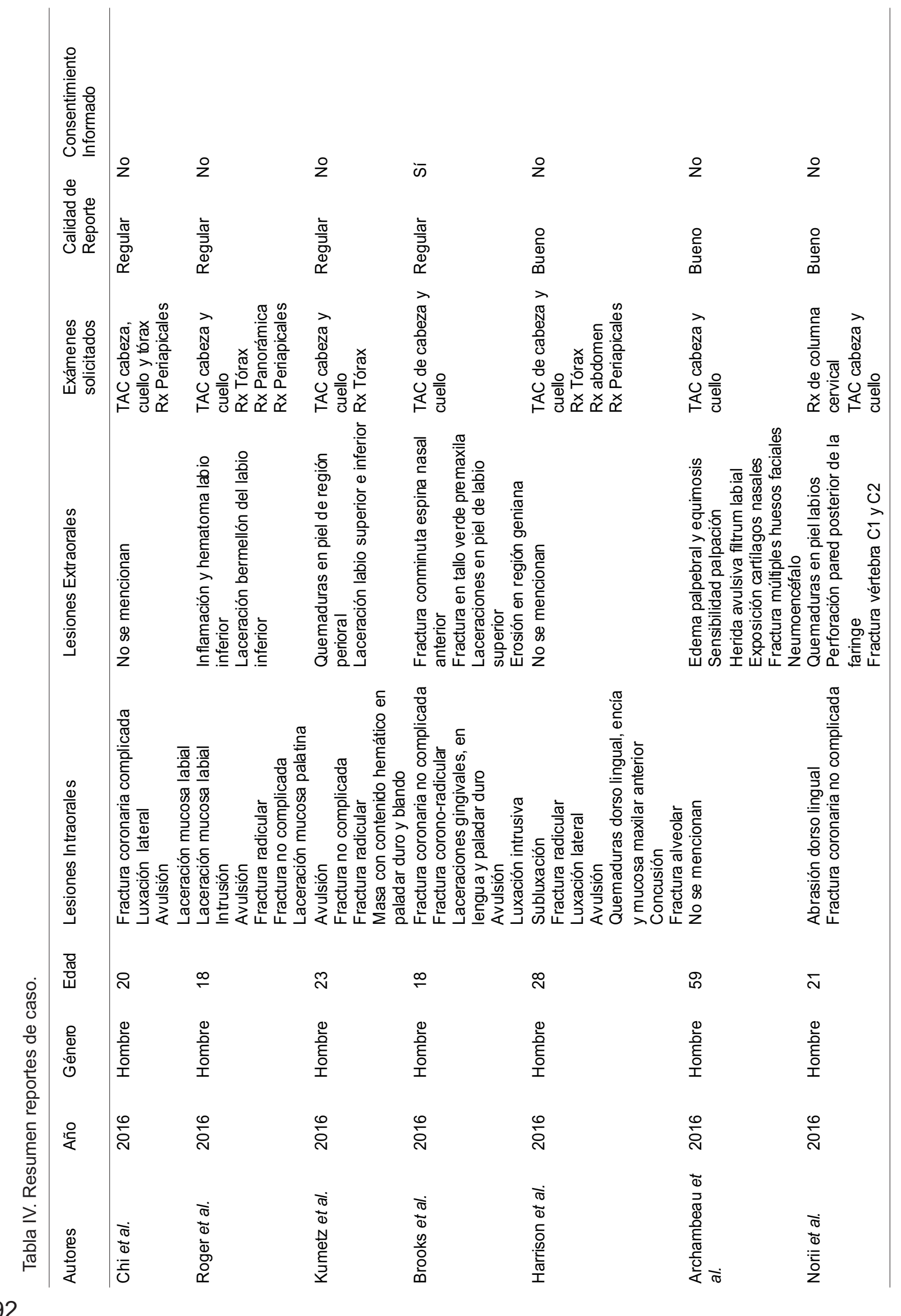


guíneo luego de utilizar el cigarro electrónico con un láser Doppler y evidenció que había cambios en la perfusión sanguínea en el grupo con y sin nicotina, siendo más marcada la disminución del flujo en aquellos con nicotina. Los resultados presentaron alta variabilidad y por lo tanto, el estudio no fue concluyente. Papaseit et al. (2017) tomaron muestras de sangre y saliva luego de utilizar un cigarro electrónico con nicotina $0,8 \mathrm{mg}$ y cigarro convencional. Se observó que las concentraciones de cotinina y nicotina en saliva, para ambos grupos, eran 45 veces más altas que en sangre y la cantidad de nicotina y sus metabolitos, eran siempre mayores para el grupo de tabaco. Así se establece que la concentración alcanzada en sangre y en saliva es dependiente de la inhalación (duración y profundidad de ésta) para ambos grupos. Entonces, aunque el cigarro electrónico tenga efectos menos marcados que el tabaco a nivel de los tejidos, igual alcanza altas concentraciones de nicotina y sus metabolitos a nivel sanguíneo y salival, afectando el flujo sanguíneo y acumulándose en los fluidos orales.

Finalmente, en cuanto a los riesgos implícitos en el uso de los dispositivos de administración electrónica de nicotina, se ha reportado en la literatura un aumento de casos de efectos adversos. La mejor evidencia disponible corresponde a reportes de caso (Tabla IV), donde el $100 \%$ de los casos ocurrió en hombres, mientras estaban utilizando el dispositivo (Kumetz et al., 2016; Brooks et al., 2017). Los pacientes incluidos, en general tenían entre 18 y 30 años. Todos los casos constituyen situaciones donde el cigarro electrónico explotó y salió proyectado, debido a un sobrecalentamiento de las baterías de litio, generando trauma a nivel de los tejidos.

El gran peligro de estas explosiones en la separación de los fragmentos, donde el dispositivo pasa a convertirse en un verdadero proyectil, que puede generar gran trauma en la región hacia donde salga disparado. Esto ocurrió en el caso de Norii \& Plate (2017), donde la mitad del cigarro electrónico quedó alojada en la pared posterior de la faringe, perforándola y causando la fractura de las vértebras $\mathrm{C} 1$ y C2. Otro caso ocurrido en un paciente de 59 años, reportado por Archambeau et al. (2016), donde producto del trauma el paciente sufrió un neumoencéfalo, el cual se resolvió un mes después. Estos casos revelan la gravedad de estas explosiones y son un reflejo de los riesgos que estos representan.
Las lesiones intraorales también son severas debido a que el trauma es de alta intensidad y hay una explosión de por medio, lo que genera quemaduras en piel, mucosa y abrasiones. En todos los casos encontrados, menos en el estudio de Archambeau et al. se produjo avulsión de alguna pieza dentaria, siendo las piezas más afectadas las anteriores, tanto superiores como inferiores, donde lo más común es la fractura coronaria.

En conclusión y como respuesta a los objetivos propuestos al principio de este trabajo, se puede establecer que el cigarro electrónico es citotóxico y potencialmente dañino para la cavidad oral en general. Con excepción de un estudio in vitro que no es concluyente, todos los demás establecen que el cigarro electrónico es tóxico y causa daños estructurales, como necrosis y apoptosis celular, disminuyendo la viabilidad de las células periodontales, específicamente células epiteliales gingivales y fibroblastos gingivales y del ligamento periodontal.

En comparación con el cigarro convencional se ha evidenciado que los dispositivos de administración electrónica de nicotina serían menos tóxicos para los tejidos periodontales, mostrando mejoras en los parámetros periodontales, como sangramiento e índice de placa bacteriana. Sin embargo, esto debe ser interpretado con cautela.

Por último, los traumatismos y eventos adversos ocurridos sobre la región orofacial dejan en evidencia los riesgos inherentes al uso del cigarro electrónico. Si bien el objetivo de esta revisión no es dar recomendaciones, el clínico debe informarse y evaluar si su uso es aconsejable.

Las limitaciones de los trabajos incluidos son claras y han sido explicitadas durante la revisión. No obstante, constituyen una primera línea de investigación y promueven que se continúe investigando con respecto al tema, buscando realizar estudios de mejor calidad, con un mayor tamaño de muestra e idealmente con un seguimiento mayor.

\section{AGRADECIMIENTOS}

A mi profesora guía en este trabajo y gestora de la idea, Dra. Paula Riera, por su constante motivación e impulso para seguir adelante con esta publicación. 
ZILLERUELO, P. M. J.; RIERA, S. P. \& GEORGUDIS, P. G. Electronic nicotine delivery systems and their effect in periodontal tissue and the orofacial region. A review. Int. J. Odontostomat., 12(3):287-295, 2018.

ABSTRACT: Electronic nicotine delivery systems were introduced to replace tobacco use and contribute to the anti-smoking policies. Today, the effect the chemicals it contains produce in the human body, are unknown. The aim of this work was to describe the information found in the literature about the possible damage produced in the periodontal tissue and the oral cavity, by the electronic nicotine delivery systems. An electronic and manual search was carried by a single reviewer, without year or language limit, excluding expert's opinions and articles that studied other nicotine delivery systems. Eighteen articles were selected; 7 in vitro studies, 2 longitudinal studies, 2 clinical trials and 7 case reports. Toxicity levels were found to be high, showing changes in cellular morphology and metabolism. Comparing with conventional cigarette, toxicity levels were lower in the electronic nicotine delivery systems. Longitudinal studies and clinical trials observed an increase in bleeding on probing and in blood circulation, by using laser doppler velocimetry, when changing from conventional cigarette to electronic cigarette. Also reports about explosions while using these electronic devises were encountered in the literature, with grave consequences in the maxillofacial territory. Electronic nicotine delivery systems show toxic levels, generating necrosis and DNA damage in periodontal ligament and gingival fibroblast cells. The risk of using these devices is high, due to possible explosion following overheating of the lithium battery, causing facial and oral trauma. Clinical trials and longitudinal studies were not conclusive, so investigations should continue in this matter.

KEY WORDS: Electronic Nicotine Delivery Systems, periodontal diseases, mouth.

\section{REFERENCIAS BIBLIOGRÁFICAS}

Archambeau, B. A.; Young, S.; Lee, C.; Pennington, T.; Vanderbeek, C.; Miulli, D.; Culhane, J. \& Neeki, M. E-cigarette Blast Injury: Complex Facial Fractures and Pneumocephalus. West J. Emerg. Med., 17(6):805-7, 2016.

Born, H.; Persky, M.; Kraus, D. H.; Peng, R.; Amin, M. R. \& Branski, R. C. Electronic cigarettes: a primer for clinicians. Otolaryngol. Head Neck Surg., 153(1):5-14, 2015.

Brooks, J. K.; Kleinman, J. W.; Brooks, J. B. \& Reynolds, M. A. Electronic cigarette explosion associated with extensive intraoral injuries. Dent. Traumatol., 33(2):149-52, 2017.

Chen, I. L. FDA summary of adverse events on electronic cigarettes. Nicotine Tob. Res., 15(2):615-6, 2013.

Ebbert, J. O.; Agunwamba, A. A. \& Rutten, L. J. Counseling patients on the use of electronic cigarettes. Mayo Clin. Proc., 90(1):128-34, 2015.
Ito, Y.; Bhawal, U. K.; Sasahira, T.; Toyama, T.; Sato, T.; Matsuda, D.; Nishikiori, H.; Kobayashi, M.; Sugiyama, M.; Hamada, N.; Arakawa, H. \& Kuniyasu, H. Involvement of HMGB1 and RAGE in IL-1b-induced gingival inflammation. Arch. Oral Biol., 57(1):73-80, 2012.

Ji, E. H.; Sun, B.; Zhao, T.; Shu, S.; Chang, C. H.; Messadi, D.; Xia, T.; Zhu, Y. \& Hu, S. Characterization of electronic cigarette aerosol and its induction of oxidative stress response in oral keratinocytes. PLoS One, 11(5):e0154447, 2016.

Kanodia, D. E- Cigarette 101. Oral Health, 10(5):26-7, 2016.

Kim, H. J. \& Shin, H. S. Determination of tobacco-specific nitrosamines in replacement liquids of electronic cigarettes by liquid chromatography-tandem mass spectrometry. $J$. Chromatogr. A, 1291:48-55, 2013.

Kumetz, E. A.; Hurst, N. D.; Cudnik, R. J. \& Rudinsky, S. L. Electronic cigarette explosion injuries. Am. J. Emerg. Med., 34(11):2252.e1-2252.e3, 2016.

Ley 20.660. Modifica Ley $N^{\circ} 19.419$, En Materia de Ambientes Libres de Humo de Tabaco. Santiago de Chile, Ministerio de Salud, Subsecretaría de Salud Pública, 2013. Disponible en: http://www.leychile.cl/ Navegar?idNorma $=1047848$ \&idVersion $=2013-03-01$

Marynak, K. L.; Gammon, D. G.; Rogers, T.; Coats, E. M.; Singh, T. \& King, B. A. Sales of nicotine-containing electronic cigarette products: United States, 2015. Am. J. Public Health, 107(5):702-5, 2017.

Norii, T. \& Plate, A. Electronic cigarette explosion resulting in a C1 and C2 fracture: a case report. J. Emerg. Med., 52(1):86-8, 2017

Papaseit, E.; Farré, M.; Graziano, S.; Pacifici, R.; Pérez-Mañá, C.; García-Algar, O. \& Pichini, S. Monitoring nicotine intake from e-cigarettes: measurement of parent drug and metabolites in oral fluid and plasma. Clin. Chem. Lab. Med., 55(3):415-23, 2017.

Ramôa, C. P.; Eissenberg, T. \& Sahingur, S. E. Increasing popularity of waterpipe tobacco smoking and electronic cigarette use: Implications for oral healthcare. J. Periodontal. Res., 52(5):813-23, 2017.

Reuther, W. J.; Hale, B.; Matharu, J.; Blythe, J. N. \& Brennan, P. A. Do you mind if I vape? Immediate effects of electronic cigarettes on perfusion in buccal mucosal tissue--a pilot study. Br. J. Oral Maxillofac. Surg., 54(3):338-41, 2016.

Rouabhia, M.; Park, H. J.; Semlali, A.; Zakrzewski, A.; Chmielewski, W. \& Chakir, J. E-cigarette vapor induces an apoptotic response in human gingival epithelial cells through the caspase-3 pathway. J. Cell. Physiol., 232(6):1539-47, 2017.

Sancilio, S.; Gallorini, M.; Cataldi, A. \& di Giacomo, V. Cytotoxicity and apoptosis induction by e-cigarette fluids in human gingival fibroblasts. Clin. Oral Investig., 20(3):477-83, 2016.

Sancilio, S.; Gallorini, M.; Cataldi, A.; Sancillo, L.; Rana, R. A. \& di Giacomo, V. Modifications in human oral fibroblast ultrastructure, collagen production, and Iysosomal compartment in response to electronic cigarette fluids. J. Periodontol., 88(7):673-80, 2017.

Sundar, I. K.; Javed, F.; Romanos, G. E. \& Rahman, I. Ecigarettes and flavorings induce inflammatory and prosenescence responses in oral epithelial cells and periodontal fibroblasts. Oncotarget, 7(47):77196-204, 2016.

Tatullo, M.; Gentile, S.; Paduano, F.; Santacroce, L. \& Marrelli, $M$. Crosstalk between oral and general health status in esmokers. Medicine (Baltimore), 95(49):e5589, 2016. 
Wadia, R.; Booth, V.; Yap, H. F. \& Moyes, D. L. A pilot study of the gingival response when smokers switch from smoking to vaping. Br. Dent. J., 221(11):722-6, 2016.

Wang, Q.; Ping, P.; Zhao, X.; Chu, G.; Sun, J. \& Chen, C. Thermal runaway caused fire and explosion of lithium ion battery. J. Power Sources, 208:210-24, 2012.

Willershausen, I.; Wolf, T.; Weyer, V.; Sader, R.; Ghanaati, S. \& Willershausen, B. Influence of E-smoking liquids on human periodontal ligament fibroblasts. Head Face Med., 10:39, 2014.

Yu, V.; Rahimy, M.; Korrapati, A.; Xuan, Y.; Zou, A. E.; Krishnan, A. R.; Tsui, T.; Aguilera, J. A.; Advani, S.; Crotty Alexander, L. E.; Brumund, K. T.; Wang-Rodriguez, J. \& Ongkeko, W. $\mathrm{M}$. Electronic cigarettes induce DNA strand breaks and cell death independently of nicotine in cell lines. Oral Oncol., 52:58-65, 2016.
Dirección para correspondencia:

María José Zilleruelo Pozo

Universidad de los Andes

Monseñor Álvaro del Portillo 12455

Las Condes

Santiago

CHILE

Email: mjosezilleruelo@gmail.com

Recibido : 08-06-2018

Aceptado: 08-07-2018 